\title{
La mortalidad por suicidio en las poblaciones masculinas joven, adulta y adulta mayor en ocho países de Latinoamérica y el Caribe
}

\section{Suicide mortality in young, adult and older male populations in eight Latin American and Caribbean countries}

\section{Rosario Cárdenas}

Orcid: 0000-0001-6586-8669

cardenas.rsr@gmail.com

Profesora-investigadora de tiempo completo en la Universidad Autónoma Metropolitana, México

\section{Resumen}

El suicidio es la manifestación más extrema de necesidades de atención de salud mental y la importancia de reducir su ocurrencia ha sido subrayada por los Objetivos de Desarrollo Sostenible. La pandemia de COVID-19 podría incrementar la mortalidad por suicidio debido a sus efectos negativos sobre las condiciones de vida. Este trabajo analiza la mortalidad por suicidio en hombres jóvenes, adultos y adultos mayores en ocho países de Latinoamérica y el Caribe entre 2005 y 2015. Se estiman tasas de mortalidad anuales para cinco grupos etarios distinguidos por etapas de vida. Los resultados muestran diferencias por países entre los patrones de edad y la trayectoria de esta causa de mortalidad. Uruguay manifiesta los niveles más altos de suicidio junto con Cuba para los adultos mayores. Hallazgos como los que este estudio y otros similares proveen respecto a la tendencia del suicidio en la región de Latinoamérica y el Caribe o los países que la integran, podrían servir de punto de referencia para valorar el impacto de la pandemia de SARS-CoV-2 para esta problemática de la salud mental.

Palabras

clave

Suicidio

Mortalidad

Salud mental

Jóvenes

Adultos

Adultos mayores

Latinoamérica

Caribe 


\begin{abstract}
Suicide is the most extreme manifestation of mental health care needs and the importance of reducing its occurrence has been underscored by the Sustainable Development Goals. The COVID-19 pandemic could increase suicide mortality due to its negative effects on living conditions. This paper analyzes suicide mortality in young men, adults, and older adults in eight Latin American and Caribbean countries between 2005 and 2015. Annual mortality rates are estimated for five age groups distinguished by life stages. The results show differences by country between age patterns and the trajectory of this cause of mortality. Uruguay shows the highest levels of suicide for all age groups analyzed along with Cuba for the elderly. Findings such as the ones reported in this study and other similar regarding the suicide trend in the Latin American and Caribbean region or the countries that comprise it, could serve as a point of reference to assess the impact of the SARS-COV-2 pandemic for this mental health problem.
\end{abstract}

Recibido: 29/09/2020 Aceptado: 17/11/2020

\title{
Introducción
}

El suicidio es la expresión más extrema de condiciones que manifiestan problemas de salud mental como la depresión (Oliffe et al., 2019), la ansiedad (Stanley et al., 2018), el abuso en el consumo de alcohol (Ghanbari et al., 2015) o las adicciones (Carmel et al., 2016; Yuodelis-Floresy Ries, 2015), pero también de circunstancias de vida estresantes como el desempleo, la inestabilidad laboral, la mala calidad del trabajo (Chastang et al., 1998; Min et al., 2015; Nordt et al., 2015), relaciones personales insatisfactorias (Till, Tran y Niederkrotenthaler, 2017), rompimientos sentimentales (Love et al., 2018) o el aislamiento social (Calati et al., 2019). Haber sufrido negligencia, abuso físico (Felitti et al., 2019) o sexual durante la infancia (Pérez-Fuentes et al., 2013; Spokas et al., 2009), estrés postraumático (Bach et al., 2018) o alteraciones del sueño (Bernert et al., 2015; Pigeon, Pinquart y Conner, 2012) también incrementan las ideaciones suicidas, los intentos de suicidio o el suicidio mismo.

Se estima que en 2016 ocurrieron alrededor de 817,000 suicidios a nivel mundial y la frecuencia con la cual acontece ubica a esta causa de muerte entre las diez primeras en varias regiones del planeta incluyendo la zona sur de Latinoamérica (Naghavi et al., 2019). La Organización Panamericana de la Salud (OPS) señala que en el continente americano cada año se registran 
65,000 suicidios, lo que equivale, para el periodo 2005-2009, a una tasa estandarizada de mortalidad de 7.3 defunciones por suicidio por 100,000 habitantes, cifra que disminuye al examinar la situación que prevalece en América Latina y el Caribe exclusivamente, con una tasa de 5.2 suicidios por 100,000 habitantes (OPS, 2014, p. 5). El conjunto de las enfermedades mentales, neurológicas, de abuso de sustancias y de suicidios representa alrededor de una quinta parte de los años de vida ajustados por discapacidad y más de un tercio de los años vividos con una discapacidad, lo que traduce el impacto de estas patologías en la salud de la población (Vigo et al., 2018). La mortalidad por suicidio tiene lugar con diferentes intensidades según sexo (Otzen et al., 2014).

La OPS estima que en 2005-2009 en América Latina y el Caribe la tasa estandarizada de mortalidad por suicidio de la población masculina fue 8.39 defunciones por 100,000 hombres, para la población femenina de 2.12 por 100,000 mujeres (OPS, 2014, p. 14) y que para la población total suceden alrededor de 4 suicidios de hombres por cada suicidio femenino, cifra que aumenta al considerar al grupo de 70 años o más con 7.8 suicidios masculinos por cada uno femenino y entre 6 y 8 suicidios masculinos por cada uno femenino al llegar a los 80 años (Martini et al., 2019; Núñez-Samudio et al., 2020; OPS, 2014, p. 19). En América Latina y el Caribe, la mortalidad por suicidio también muestra un patrón diferenciado por edad entre hombres: en el grupo de 20 a 24 años esta causa de muerte corresponde al $7 \%$ del total de las defunciones por causas externas, mientras que en la población masculina de 60 a 69 años dicha proporción es 10.8\% (OPS, 2014, p. 17).

El suicidio ha sido reconocido como una manifestación de las carencias de atención a la salud mental. Por ello, el llamado para reducir su frecuencia ha quedado integrado en la estrategia de los Objetivos de Desarrollo Sostenible (ODS). En la cual, la meta 3.4 señala como propósito promover la salud mental y el bienestar evaluando la satisfacción de estos mediante la disminución de la tasa de mortalidad por suicidio.

Los estudios registran resultados mixtos para la asociación entre crisis económicas y la mortalidad debida a suicidios. Mientras algunos análisis reportan una relación positiva, especialmente para la población masculina (Chan et al., 2014; Chang et al., 2013; Milner, Morrell y LaMontagne, 2014; Saurina et al., 2013) o quienes padecen alguna enfermedad (Merzagora et al., 2016), otros no identifican que este sea el caso (Laanani et al., 2015). Elementos como la fortaleza de los sistemas de protección social parecieran explicar la ausencia de un efecto de crisis económicas severas, como la que tuvo lugar a nivel mundial en 2008, en la mortalidad por suicidio (Ásgeirsdóttir et al., 2020; Van Hal, 2015). 
La pandemia de COVID-19, provocada por el virus SARS-CoV-2, ha desencadenado no solo una crisis sanitaria sino también una de índole económica cuya envergadura está aún por precisarse dado que esta no ha sido controlada en la mayor parte de los países donde esta enfermedad se ha registrado. En el caso de Latinoamérica y el Caribe, la Comisión Económica para América Latina y el Caribe (CEPAL, 2020) estima que el impacto de la pandemia de COVID-19 provocará en 2020 una reducción del producto interno bruto (PIB) de -9.1\%, lo que representará un retroceso de una década en el ingreso de los habitantes de los 33 países que integran esta región con el consecuente aumento de la proporción de población en situación de pobreza y el ahondamiento de la desigualdad económica registradas en esta zona del mundo.

Con lo anterior, aunado a la debilidad de los sistemas de protección social de varios de los países (Cecchini, Filgueira y Robles, 2014), se prevé que el efecto de la pandemia de COVID-19 será de gran magnitud y profundizará las condiciones adversas en las cuales se encuentran amplios conjuntos de las poblaciones latinoamericanas y caribeñas debido, entre otros factores, a la mortalidad de integrantes de la familia, la pérdida de empleos, el deterioro de ingresos y la afectación al acceso a los procesos educativos. Por otra parte, algunos análisis muestran, como consecuencia del aislamiento social asociado a la pandemia, la recaída o aumento de adicciones del tipo del abuso en el uso de alcohol o tabaquismo (Håkansson, 2020; Sun et al., 2020) y posiblemente también a otras sustancias como heroína o cocaína, luego de haberse interrumpido los servicios de apoyo (Dubey et al., 2020), incrementando con ello el riesgo de intentos de suicidio o de suicidio.

El examen de la mortalidad por suicidio permite reconocer las carencias en torno a la salud mental presentes en una población, así como el impulso que diversos factores sociales y económicos pueden proveer para su ocurrencia. El estudio de la intensidad de la mortalidad debida a suicidio, sus diferenciales por edad, patrones de las trayectorias que describe en el tiempo y el contraste entre poblaciones permite elaborar un diagnóstico de la severidad de la situación que guarda esta causa de defunción prevenible, además que es posible identificar los grupos que con mayor urgencia requieren el diseño de acciones de política pública. Frente a la pandemia de COVID-19, las estimaciones sobre mortalidad por suicidio para años recientes sirven, además, como punto de referencia para evaluar el impacto que esta crisis sanitaria podrá tener en un área especialmente sensible como el de salud mental. 


\section{Objetivo}

El objetivo de este trabajo es analizar la tendencia de la mortalidad por suicidio en el periodo de 2005-2015. Para este efecto, se dispone de información comparable de ese periodo para las poblaciones masculinas joven, adulta y adulta mayor de ocho naciones de Latinoamérica y el Caribe. El diagnóstico de la situación de salud mental que prevalece en estos Estados, cuya aproximación ha sido mediante el estudio de la mortalidad debida a suicidio, sus diferencias para los grupos de edad estudiados, los patrones de ocurrencia de estas autolesiones y el contraste entre países, propicia distinguir las necesidades en materia de prevención y el tipo de acciones de política pública requeridas para eliminar esta causa de mortalidad prevenible.

\section{Material y métodos}

La información sobre mortalidad utilizada para la realización de este trabajo proviene de las bases de datos por causas específicas de la Organización Mundial de la Salud (World Health Organization [WHO], 202O). Los años de estudio fueron definidos en función de la disponibilidad de información sobre mortalidad para los países analizados tomando como punto de partida el más reciente reportado, 2015. Para Uruguay la información sobre mortalidad de 2011 no está aún registrada en estas bases de datos, tampoco se encuentra disponible con el nivel de detalle requerido (edad, sexo y causa específica de muerte) en la página web del Instituto Nacional de Estadística de Uruguay. El suicidio es una causa de muerte cuyo registro se ve afectado por aspectos culturales y religiosos, así como por estigmas sobre su realización, influyendo con ello en la completitud de los datos (Mascayano et al., 2015, 2016; Pritchard y Hean, 2008). Adicional a lo anterior, el denominado efecto Werther, es decir, cuando el suicidio de una figura pública, frecuentemente alguien vinculada con un medio artístico, conlleva un aumento en el número de suicidios, se asocia también a fluctuaciones en su ocurrencia (Fahey et al., 2018).

La comparación de varios años calendario posibilita distinguir el patrón de ocurrencia de la mortalidad por suicidio, soslayando las variaciones que los factores antes señalados pudieran haber provocado en el acaecimiento de estos o en su registro. Examinar un periodo de poco más de una década, 2005-2015, permite recuperar los patrones de comportamiento de esta causa de muerte. El conjunto de países estudiados está integrado por ocho naciones: Argentina, Brasil, Chile, Colombia, Cuba, Ecuador, México y Uruguay, los cuales fueron incorporados con base en dos criterios: la 
disponibilidad de información para los años señalados para el estudio y que la intensidad de la mortalidad por suicidio posibilitara la obtención de tasas de mortalidad anuales para los grupos etarios de interés con numeradores que al menos considerarán 30 defunciones. Si bien la selección de los países que conforman el conjunto analizado responde a los dos elementos antes expuestos, la inclusión de Argentina, Chile, Cuba y Uruguay, naciones que registran altas tasas de mortalidad por suicidio en hombres en América Latina y el Caribe, contribuye a tener una imagen más completa de la situación en la región. Un estudio de la OPS para el quinquenio 2005-2009 reportó tasas de mortalidad en estos países de 12.56, 19.27, 19.05 y 26.01 defunciones por 100,000 hombres, respectivamente, cifras que contrastan con las estimadas para Guatemala y Perú (3.44 y 1.29) (OPS, 2014, p. 52-53).

Debido a las diferencias por sexo en los niveles y por grupo de edad de esta causa específica de defunción, previamente mencionadas este trabajo, se ha optado abordar únicamente la mortalidad masculina por suicidio. El estudio se concentra en identificar los niveles, tendencias y pautas de ocurrencia por edad de la población de 20 años o más. Si bien en los países estudiados se reportan casos de suicidio entre la población adolescente, su estudio requiere del cálculo de tasas multianuales, el cual difiere de la aproximación empleada en este análisis que evidencia los cambios anuales en la intensidad de la mortalidad por suicidio entre 2005 y 2015.

Las edades estudiadas han sido agrupadas para reflejar la gravedad de esta problemática social y de salud de acuerdo a etapas de la vida. Los grupos de edad definidos son: de 20 a 29, 30 a 44, 45 a 59, 60 a 74 y 75 años o más. La información poblacional empleada como numerador para la estimación de las tasas de mortalidad es la producida por el Departamento de Asuntos Económicos y Sociales de Naciones Unidas en su ejercicio de prospectiva de la población 2019 (United Nations, 2020b). Las edades que Naciones Unidas publica en grupos de cinco años fueron categorizadas para este estudio de acuerdo con lo señalado respecto a los grupos etarios de estudio. Las tasas de mortalidad se presentan expresadas por 100,000 habitantes del grupo de edad correspondiente. Las defunciones debidas a suicidio fueron identificadas mediante los códigos X60 a X84 de la décima revisión de la Clasificación Internacional Estadística de Enfermedades y Problemas de Salud Relacionados (ICD-10, 2019). Con el propósito de evidenciar la posible sobremortalidad entre países, se estimó el cociente entre las tasas de mortalidad observadas y una tasa de mortalidad de una de las naciones analizadas, la cual fue empleada como referencia. Cabe señalar que esta nación referencial, utilizada para cada grupo de edad, es la que registró la serie con las menores tasas de mortalidad por suicidio durante el periodo. 


\section{Resultados}

La Gráfica 1 presenta las tasas de mortalidad masculina de la población de 20 a 29 años debida a suicidio, expresadas por 100,000 hombres de dicho grupo etario. Esta información revela que entre 2005 y 2015 los diferenciales en las tasas de mortalidad entre los países analizados se redujeron, con excepción de Uruguay que muestra una clara tendencia al aumento durante este periodo. También indica que Chile revirtió el incremento registrado en la mortalidad por esta causa específica entre 2007 y 2009 mostrando para 2015 una tasa menor que la observada en 2005. Brasil, Colombia, Cuba y México mantuvieron durante el periodo estudiado las tasas más bajas de mortalidad por suicidio para la población de hombres jóvenes, además de una estabilidad en la intensidad con la cual sobreviene este fenómeno. Argentina muestra un patrón similar al de los cuatro países antes mencionados en cuanto a la persistencia de los niveles de mortalidad por suicidio de hombres jóvenes (20 a 29 años), pero con tasas que durante todo ese periodo esencialmente duplican las estimadas para Brasil. Si bien Ecuador mantiene una tendencia decreciente para las tasas de mortalidad por suicidio de hombres jóvenes para la mayoría de los años que integran la serie analizada, la estimación correspondiente a 2015 tiene un valor cercano al observado en 2005 , lo cual evidencia la importancia de darle seguimiento cercano a la evolución de la mortalidad por suicidio en esta nación, al igual que en el caso de Uruguay.

Gráfica 1. Tasas de mortalidad de la población masculina de 20 a 29 años debida a suicidio (por 100,000), para ocho países de Latinoamérica y el Caribe, 2005-2015

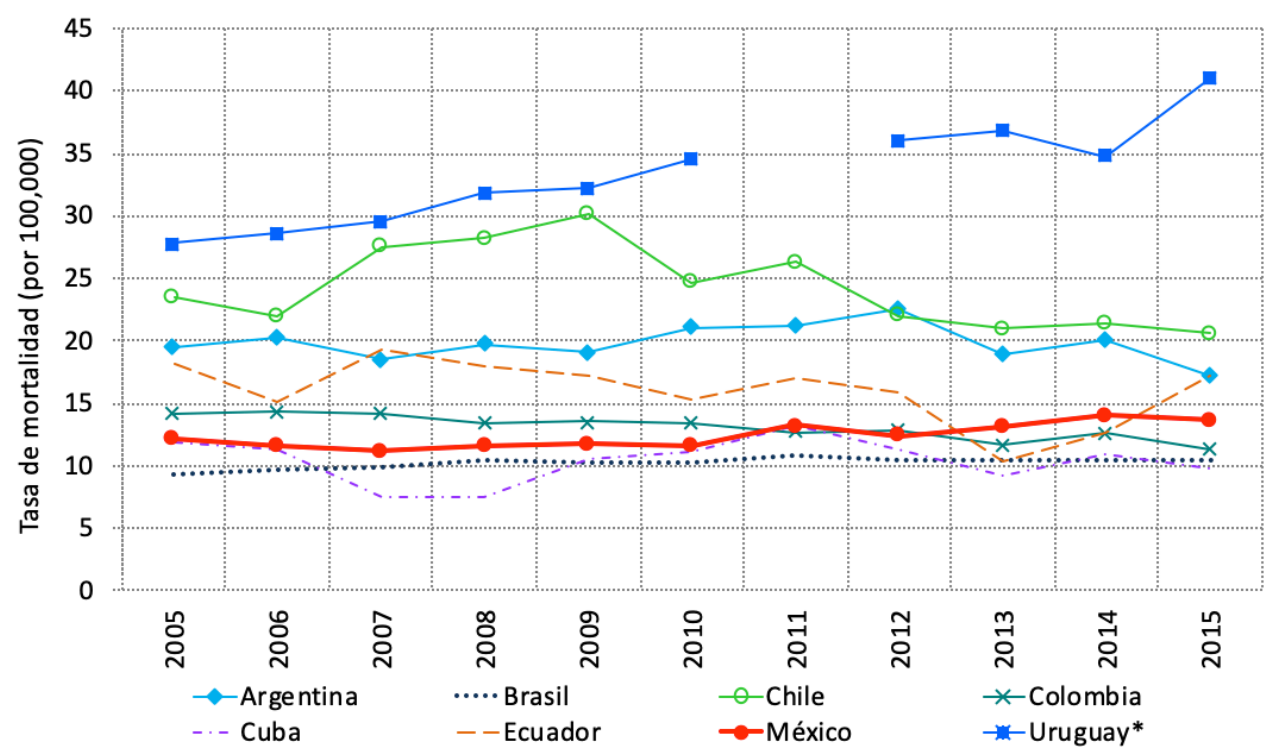

*Para 2017 no hay información disponible sobre mortalidad para Uruguay.

Fuente: elaboración propia a partir de WHO Mortality Database y estimaciones de población de Naciones Unidas. 
La Tabla 1 muestra la comparación entre las tasas específicas de mortalidad por suicidio y grupo de edad entre países mediante el cociente que contrasta tasa de interés/tasa de referencia. Para el grupo de edad 20 a 29 años, Brasil es el país de referencia utilizado. Los resultados indican, en 2005, que el nivel de mortalidad de Cuba y México excede el observado en Brasil en 25\%, el de Colombia en 50\%, que Argentina y Ecuador esencialmente duplican la mortalidad brasileña, que la de Chile es 1.5 veces mayor y la de Uruguay es de casi el triple.

Para 2015 las desigualdades de seis de los siete países han disminuido. La única discrepancia que aumenta durante la década es la de Uruguay, cuya mortalidad por suicidio en la población joven representa casi cuatro veces la identificada en Brasil (Tabla 1).

El análisis de las tasas de mortalidad por suicidio de la población masculina adulta de 30 a 44 años indica que las tendencias de Argentina, Brasil, Colombia, Cuba, Ecuador y México se han mantenido estables entre 2005y 2015 (Gráfica 2). Entre estos países destaca que Cuba exhibe los niveles más altos de mortalidad en la población masculina de 30 a 44 años.

Gráfica 2. Tasas de mortalidad de la población masculina de 30 a 44 años debida a suicidio (por 100,000), para ocho países de Latinoamérica y el Caribe, 2005-2015

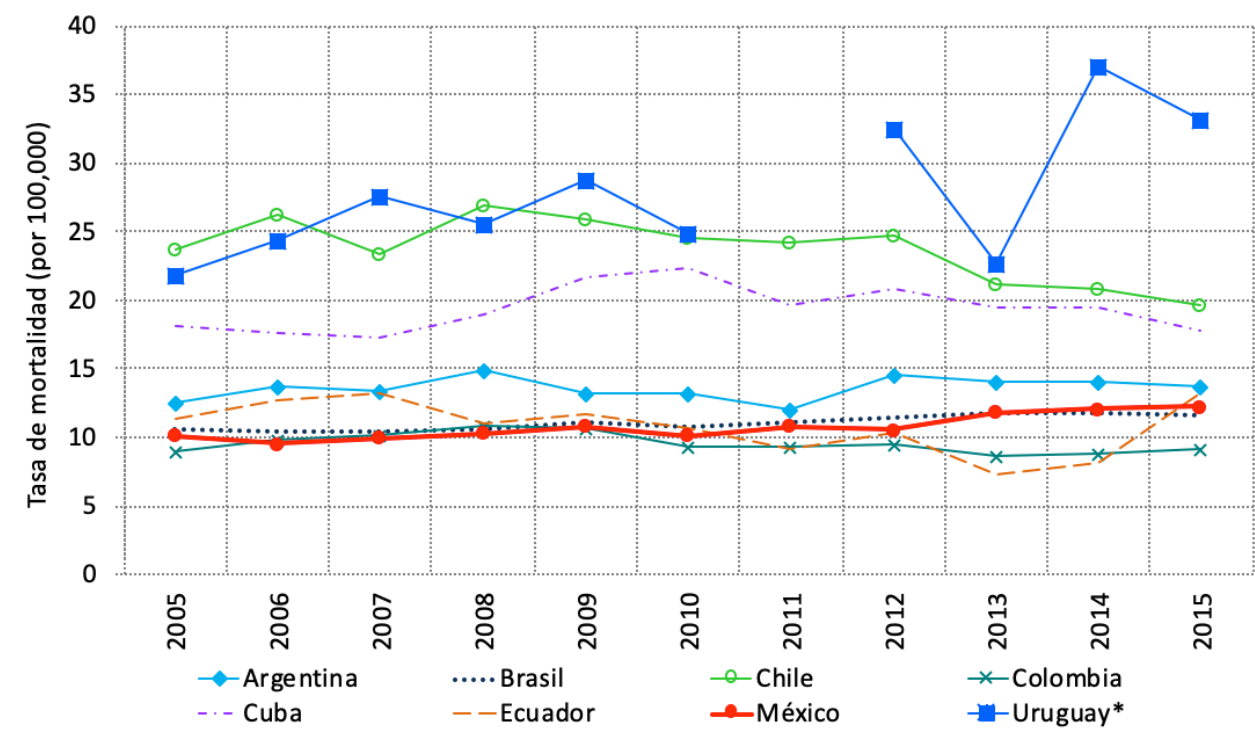

*Para 2011 no hay información disponible sobre mortalidad para Uruguay. Fuente: elaboración propia a partir de WHO Mortality Database y estimaciones de población de Naciones Unidas. 


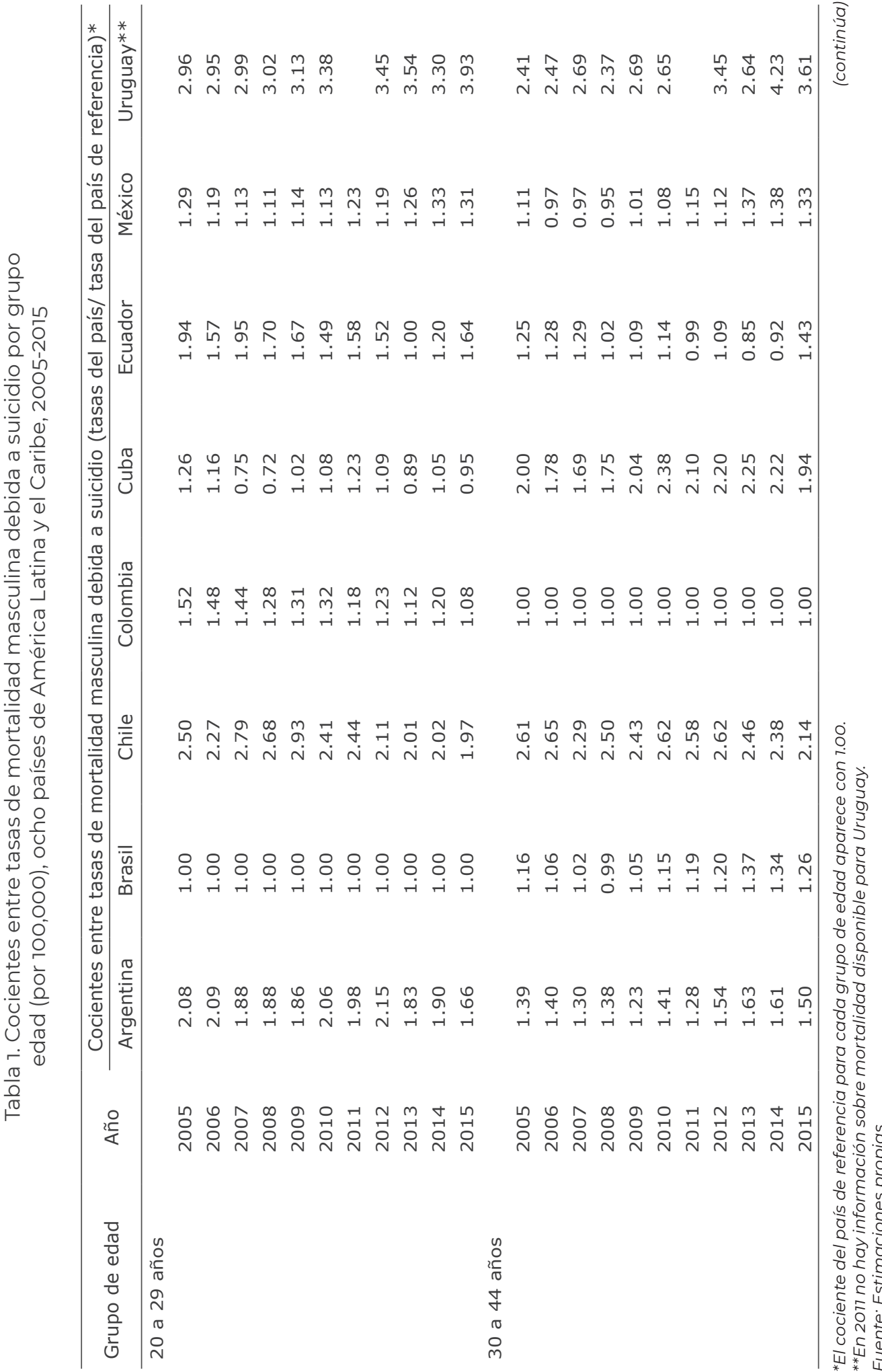



今 今

율ำ

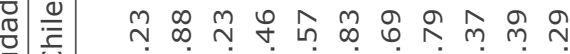

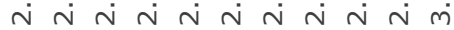

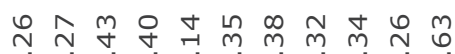
r 
Chile nuevamente manifiesta el segundo nivel más alto de mortalidad por esta causa para este grupo de adultos, sin embargo, entre 2005 y 2015 exterioriza una disminución ostensible de la intensidad de la mortalidad por suicidio. El examen de la información para este grupo etario indica que Uruguay es el país con la mayor mortalidad por suicidio en la primera etapa de la adultez (30 a 44 años). Al igual que en el caso de la población de 20 a 29 años, en 2015 Ecuador registra un incremento en el nivel de la mortalidad por esta causa, lo que sugiere la necesidad de dar seguimiento cercano al patrón que describirá en años subsecuentes.

En el segundo panel de la Tabla 1 se presentan las estimaciones comparativas de los niveles de mortalidad por suicidio entre países para el grupo de hombres de 30 a 44 años. La nación de referencia para esta agrupación es Colombia.

Los cocientes de las tasas de mortalidad indican que, en 2005, la mortalidad por suicidio en Argentina, Brasil, Ecuador y México excedían entre 10\% y casi 40\% la observada en Colombia para la población masculina de 30 a 44 años, que Cuba duplicaba la intensidad de la mortalidad por esta causa de defunción respecto a Colombia y, finalmente, que Uruguay y Chile mostraban niveles de suicidio entre 1.4 y 1.6 veces el identificado para Colombia. Para 2015, Uruguay presenta el cambio más preocupante con un incremento de la mortalidad por suicidio en hombres de 30 a 44 años que representa 3.6 veces la intensidad registrada para Colombia en el mismo año (Tabla 1).

La estimación de las tasas de mortalidad por suicidio para la población masculina de 45 a 59 años de los ocho países estudiados permite distinguir tres patrones de comportamiento durante el periodo 2005-2015. Argentina, Brasil, Colombia, Ecuador y México conforman el primer patrón con niveles de mortalidad en la parte baja de la escala con tasas entre 5 y 15 defunciones debidas a suicidio por cada 100,000 hombres de 45 a 59 años, este se sostiene durante los años analizados (Gráfica 3).

Adicionalmente, como se observa en la Gráfica 3, las diferencias entre la intensidad de la mortalidad por suicidio de los cinco países se estrechan entre 2005 y 2015. Chile presenta el segundo patrón de comportamiento para el periodo examinado con una mortalidad masculina por suicidio en los hombres de 45 a 59 años que, aunque mayor que la de los países anteriores (25 defunciones debidas a suicidios por 100,000 hombres de 45 a 59 años en 2005), se reduce levemente entre 2005 y 2015. El tercer patrón corresponde al trazo que describen las trayectorias de la mortalidad por suicidio en hombres de 45 a 59 años para Cuba y Uruguay con francos incrementos de las tasas de mortalidad entre 2005 y 2015. Al igual que para los grupos de edad previamente descritos, Uruguay registra la situación 
más apremiante dada la tendencia al aumento de la tasa de mortalidad masculina por suicidios (Gráfica 3). El tercer panel de la Tabla 1 muestra la comparación de los niveles de mortalidad masculina por suicidio entre los ocho países estudiados para el grupo de edad de 45 a 59 años. México es utilizado como referencia para este grupo etario al ser el país con el patrón de tasas de mortalidad por suicidio más bajas.

Gráfica 3. Tasas de mortalidad de la población masculina de 45 a 59 años debida a suicidio (por 100,000), para ocho países de Latinoamérica y el Caribe, 2005-2015

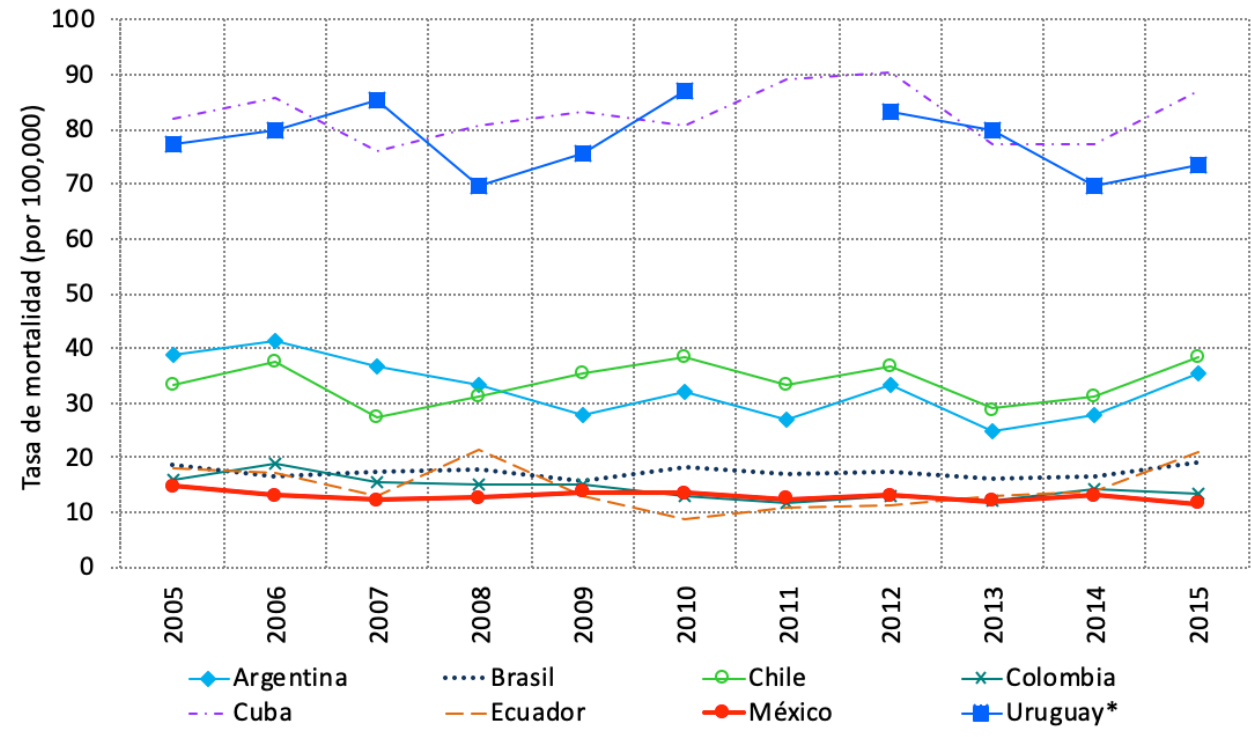

*Para 2011 no hay información disponible sobre mortalidad para Uruguay.

Fuente: elaboración propia a partir de WHO Mortality Database y estimaciones de población de Naciones Unidas.

Los resultados señalan que Colombia es el segundo país con los niveles más bajos de mortalidad por suicidio para los hombres en la etapa de la adultez que abarca de los 45 a los 59 años, cuya intensidad es alrededor de 10\% superior que la registrada en México, seguido de Brasil que presenta una mortalidad 35\% mayor que la mexicana. Al inicio del periodo estudiado, 2005, Argentina y Ecuador excedían en casi $80 \%$ la mortalidad por suicidio reportada para México, sin embargo, estos niveles descendieron durante la década examinada: para 2015 la mortalidad argentina excede poco menos de 40\% la de México y la de Ecuador, a partir de 2013, es visiblemente menor que la mexicana. Al igual que para los grupos etarios previamente descritos, los resultados de la mortalidad por suicidio de los adultos de 45 a 59 años señalan que Chile, Cuba y Uruguay son los países para los cuales se observan las mayores desigualdades respecto a sus pares considerados en este análisis. La mortalidad por suicidio de los adultos de 45 a 59 años 
en Chile en 2005 es casi el triple de la de México y, aunque disminuyó en la mayoría de los años intermedios, en 2015 continúa siendo superior en un 45\%. La mortalidad de Cuba para este grupo de edad representa, en todos los años incluidos en el análisis, esencialmente el triple de la observada en México; la de Uruguay es casi cuatro veces más alta y en 2007 quintuplicó el valor empleado como referencia.

La Gráfica 4 muestra los valores de las tasas de mortalidad masculina por suicidio para la población de 60 a 74 años de edad. Esta información permite distinguir a Brasil, Colombia, Ecuador y México como los países con la menor intensidad de suicidio entre los ochos estudiados en esta etapa de la adultez mayor. Los datos indican, además, la persistencia de la frecuencia con la cual los hombres de 60 a 74 años se suicidaron durante 2005 y 2015 en estos cuatro países. Las estimaciones de mortalidad por suicidio para Argentina y Chile revelan una tendencia decreciente de la asiduidad con la que acaecieron los suicidios en esa década. En contraste, el suicidio de este grupo etario aumentó en Cuba en ese periodo (34.9 y 39.5 suicidios por 100,000, respectivamente), alcanzando el mayor valor en 2013 (43 suicidios por 100,000). Uruguay muestra el patrón con mayores fluctuaciones en el trascurso de esta década, no obstante, la tasa de mortalidad en 2005 y 2015 fue la misma: 50 defunciones masculinas por suicidio por 100,000 adultos mayores de 60 a 74 años.

Gráfica 4. Tasas de mortalidad de la población masculina de 60 a 74 años debida a suicidio (por 100,000), para ocho países de Latinoamérica y el Caribe, 2005-2015

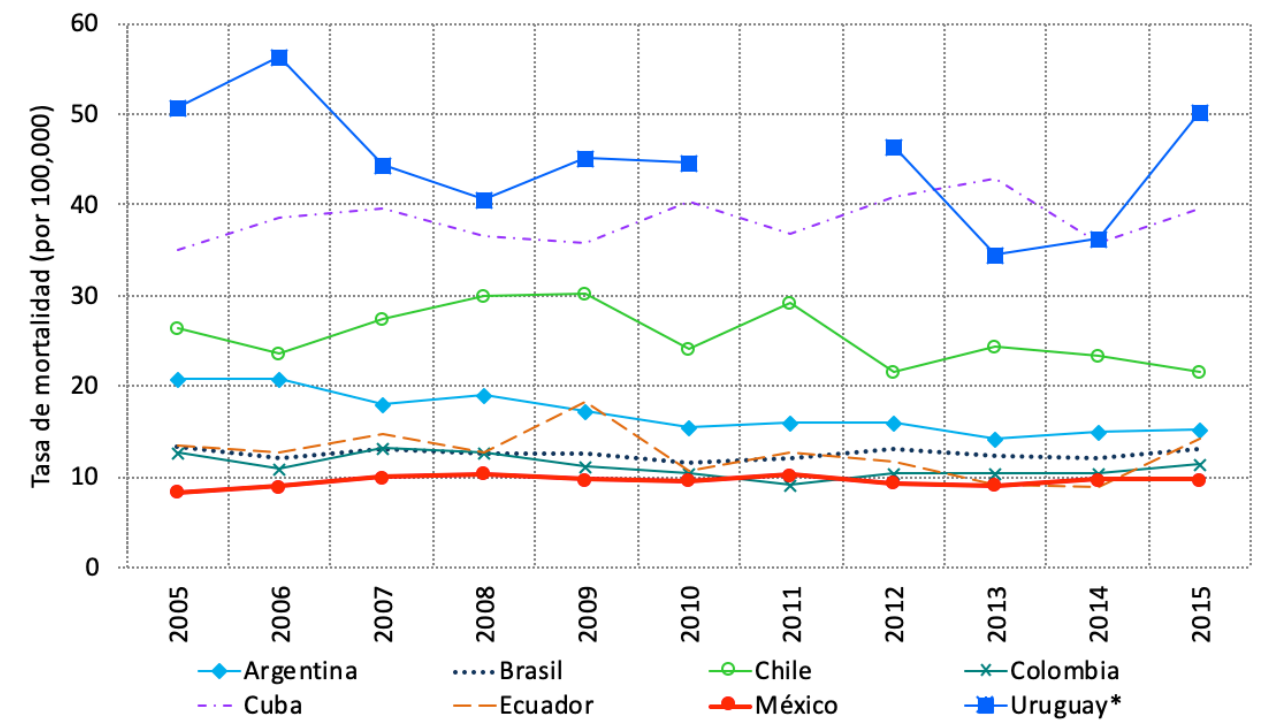

* Para 2017 no hay información disponible sobre mortalidad para Uruguay.

Fuente: elaboración propia a partir de WHO Mortality Database y estimaciones de población de Naciones Unidas. 
El cuarto segmento de la Tabla 1 despliega los resultados de las comparaciones entre las tasas de mortalidad anuales y las empleadas como referencia para el grupo de 60 a 74 años de edad. El país con los menores niveles de mortalidad por suicidio para la población masculina de este grupo etario es México, de aquí que haya sido empleado como referencia. La información indica que Brasil, Colombia y Ecuador son los países con experiencias de mortalidad más cercanas en su intensidad a la mexicana. Sin embargo, en 2005 el grado de suicidios en estos tres países para los hombres de 60 a 74 años excedía en más del 50\% la experiencia de México, mientras que para 2015 mostró una disminución ubicándose en 30\%, 17\% y $47 \%$ superiores, respectivamente.

Entre 2005 y 2015 la mortalidad masculina por suicidio en Argentina para el grupo de edad de 60 a 74 años representó entre 1.5 y 2.5 veces la observada en México, mientras la experiencia de Chile indica tasas de mortalidad más altas que, en 2005, triplicaban la mexicana y en 2015 representaban poco más del doble. Cuba y Uruguay son los países con las intensidades de suicidio de la población masculina de 60 y 74 años más altas entre el conjunto de países analizados. En el caso de Cuba, el registro de la mortalidad para esta causa señala que el suicidio ocurría, tanto en 2005 como en 2015, con una frecuencia cuatro veces más alta que en México y para Uruguay las cifras eran seis veces superiores en 2005, mientras que quintuplicaban el valor referencia en 2015 (Tabla 1).

La Gráfica 5 expone las tasas de mortalidad por suicidio de la población masculina de 75 años o más durante el periodo 2005-2015 para los ocho países analizados. De esta se desprenden tres patrones de comportamiento. Primero, las tasas más bajas de mortalidad se registran en Brasil, Colombia, Ecuador y México con fluctuaciones entre 11 y 21 suicidios por 100,000 hombres de 75 años o más. La segunda intensidad de mortalidad por suicidio se observa en Argentina y Chile, sin embargo, los patrones entre estos países difieren pues en tanto el nivel de la mortalidad argentina en 2005 y 2015 es muy similar con una leve reducción para el último año, Chile presenta un incremento al pasar de 33.2 suicidios por 100,000 hombres de 75 años o más en 2005 a 38.5 en 2015.

Al igual que para población de menor edad, las mortalidades por suicidio más altas de este grupo etario se identifican en Cuba y Uruguay. La pauta mostrada por Uruguay para la mortalidad por suicidio para esta agrupación indica fluctuaciones importantes, no obstante, la tasa de 2015 es menor a 
la del inicio de periodo en 2005 (73.4y 77.5, respectivamente). En el caso de Cuba, los resultados obtenidos señalan que la mortalidad por suicidio tendió a aumentar durante el periodo examinado, llegando al punto máximo en 2012 con una tasa de 90.4 suicidios por 100,000 hombres de 75 años o más, asimismo, revelan la mortalidad más intensa por esta causa entre el conjunto de los países estudiados.

Gráfica 5. Tasas de mortalidad de la población masculina de 75 o más años debida a suicidio (por 100,000), para ocho países de Latinoamérica y el Caribe, 2005-2015

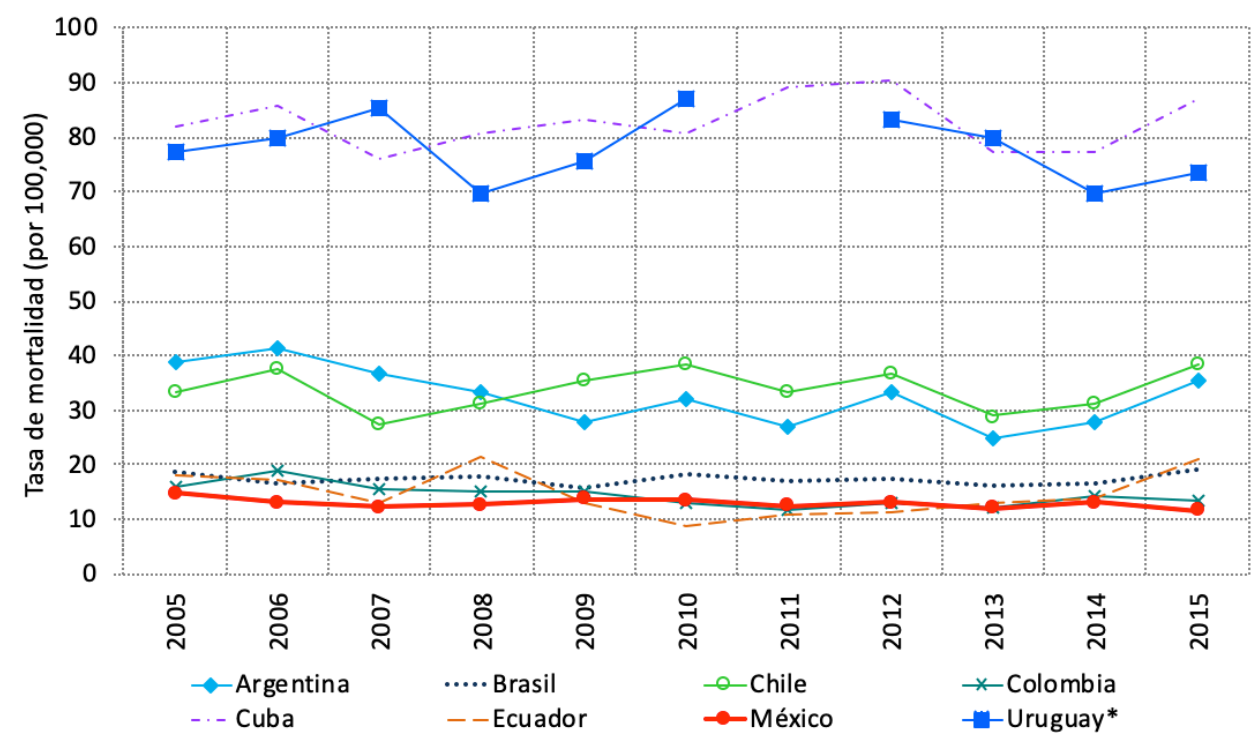

*Para 2011 no hay información disponible sobre mortalidad para Uruguay.

Fuente: elaboración propia a partir de WHO Mortality Database y estimaciones de población de Naciones Unidas.

Cabe volver a mencionar la importancia de analizar varios años a fin de recuperar de mejor forma la experiencia de la población en torno a esta problemática social y de salud pública. Ecuador ilustra las dificultades de derivar una conclusión acerca del suicidio al considerar años aislados. Para 2005 la tasa de mortalidad observada es de 18 suicidios por 100,000 hombres de 75 años o más, cifra que se redujo a 12.9 en 2007, aumentó a 21.4 en 2008, volviendo a disminuir en 2010 a 8.9 y siendo, finalmente, de 21.2 en 2015.

El quinto panel de la Tabla 1 presenta las estimaciones de la comparación entre las tasas anuales de mortalidad por suicidio para cada país y una nación de referencia, que para este grupo de edad es México al haber registrado el patrón de mortalidad más bajo. Los resultados de este contraste señalan a Colombia como el segundo país con los menores niveles de mortalidad por suicidio entre hombres de 75 años o más. Brasil y Ecuador 
describen intensidades similares de suicidio con mortalidades que exceden en 2005 alrededor de $20 \%$ la observada en México. Los niveles de las tasas de suicidios en Argentina y Chile duplican en 2005 lo encontrado para México y, para 2015, representan cifras más de tres veces superiores. Tanto Uruguay como Cuba muestran valores de mortalidad por suicidio que quintuplican lo identificado para México en 2005. Las experiencias de Cuba y Uruguay respecto al suicidio se hacen más extremas conforme avanza el periodo examinado: en 2015 las cifras de sus tasas exceden en más de seis veces las de México para el caso de Uruguay y en más de siete veces las de Cuba.

\section{Discusión}

El suicidio es un grave problema social y de salud pública, altamente susceptible de ser prevenido. Uno de los enfoques de mayor relevancia para su prevención exige reconocer que su ocurrencia es resultado de una carencia de suficientes apoyos a la salud mental en la sociedad. Las intervenciones diseñadas para identificar tempranamente las ideaciones suicidas (Klonsky, May y Saffer, 2016) y propiciar el acceso a redes de apoyo eficientes son uno de los pasos centrales que las sociedades deben emprender en la intención de reducir la frecuencia con la cual tienen lugar los suicidios. Para el caso de América Latina y el Caribe se ha reportado que el riesgo de intentar suicidarse está asociado a depresión, disfunción familiar y antecedentes de intentos previos (Teti et al., 2014); asimismo, se sabe que más de una tercera parte de quienes padecen una psicosis no afectiva, más de la mitad de quienes padecen un trastorno de ansiedad y alrededor del $75 \%$ que muestran abuso en el consumo de alcohol o una adicción no reciben atención mental (Kohn et al., 2005). Asimismo, es importante considerar que los hombres tienen más renuencia a demandar servicios de salud mental, así como a referir sintomatología de índole emocional dificultando con ello la labor de los servicios médicos, subrayando de igual manera la exigencia de implementar propuestas de atención con perspectiva de género (Oliffe et al., 2019; Rice et al., 2020).

Los hombres utilizan con mayor frecuencia armas e instrumentos para provocar asfixia (Bustamante et al., 2016; Cendales et al., 2007) como métodos para suicidarse. La Tabla 2 muestra la importancia relativa de la utilización de armas de fuego o de mecanismos que provocan asfixia como métodos para suicidarse en los ochos países analizados. Esta información refuerza la importancia de restringir la disponibilidad de armas de fuego en la sociedad (Bachmann, 2018; Mann y Michel, 2016) toda vez que en Argentina, Colombia y 
Uruguay más de una cuarta parte del total de suicidios masculinos ocurrieron como resultado del uso de estas. Los datos contenidos en la Tabla 2 también permiten reconocer el espacio del suicidio como causa de muerte tanto en su total como en el conjunto de causas externas. En Chile, Cuba y Uruguay más del $25 \%$ del total de defunciones debidas a lesiones o accidentes, es decir a causas externas, corresponde a suicidio.

Uno de los aspectos que en ocasiones no es advertido al planear acciones para reducir las ideaciones suicidas, los intentos de suicidio o el suicidio mismo es el papel que desempeñan las enfermedades crónicas y sus efectos, incluyendo dolores o amputaciones, como mecanismos que detonan los ciclos que pueden culminar en el fallecimiento por una autolesión (Arias Vázquez et al., 2018). Afectaciones para la vida sexual, como la disfunción eréctil (Klaassen et al., 2018), formar parte de una minoría sexual (Plöderl et al., 2013; Pompili et al., 2014), padecer cáncer (Fang et al., 2012; OsazuwaPeters et al., 2018), dolores crónicos (Tang y Crane, 2006) o alteraciones del sueño (Owen-Smith et al., 2019) o diabetes (Gómez-Peralta et al., 2018) han sido asociados con un aumento en el riesgo de suicidio.

Tabla 2. Distribución relativa del método empleado para suicidarse y participación relativa de los suicidios en el total de la mortalidad y la mortalidad por causas externas, población masculina, ocho países de Latino América y el Caribe, 2005-2009

\begin{tabular}{lccccc}
\hline \multirow{2}{*}{ País } & \multicolumn{2}{c}{$\begin{array}{c}\text { Método empleado para suicidarse } \\
\text { (porcentaje del total) }\end{array}$} & \multicolumn{2}{c}{$\begin{array}{c}\text { Participación de suicidios en } \\
\text { la mortalidad (porcentaje) }\end{array}$} \\
\cline { 2 - 6 } & Asfixia & $\begin{array}{c}\text { Arma de } \\
\text { fuego }\end{array}$ & $\begin{array}{c}\text { Todos los } \\
\text { demás }\end{array}$ & Total & $\begin{array}{c}\text { Causas } \\
\text { externas }\end{array}$ \\
\hline Argentina & 65.2 & 26.2 & 8.6 & 1.7 & 17.2 \\
Brasil & 62.1 & 14.4 & 23.5 & 1.3 & 6.5 \\
Chile & 83.2 & 8.0 & 8.8 & 3.4 & 25.3 \\
Colombia & 42.9 & 28.7 & 28.4 & 1.6 & 6.1 \\
Cuba & 81.9 & 2.9 & 15.2 & 2.4 & 25.4 \\
Ecuador & 53.9 & 9.2 & 36.9 & 2.3 & 9.5 \\
México & 76.3 & 14.2 & 9.5 & 1.3 & 8.2 \\
Uruguay & 59.6 & 31.4 & 9.0 & 2.8 & 29.1 \\
\hline
\end{tabular}

Fuente: Elaboración propia a partir de Organización Panamericana de la Salud (2014).

El suicidio representa también una pérdida económica para la sociedad. Un estudio llevado a cabo en Ecuador estima que durante un periodo de quince años el suicidio constituyó para este país una pérdida de 852.6 millones de dólares (Ortiz-Prado et al., 2017). 
Acciones específicas orientadas a reducir el impacto en las poblaciones que se sitúan en condiciones de particular fragilidad son indispensables como medidas para reducir la ocurrencia de suicidios (Chan et al., 2014). Los análisis que distinguen a las poblaciones indígenas u originarias, por ejemplo, identifican riesgos mayores de suicidio que sus contrapartes no indígenas (Orellana et al., 2016).

Entre las inversiones que los Estados pueden emprender para atenuar el impacto de las crisis, y con ello mantener la salud mental de sus poblaciones y disminuir la mortalidad por suicidio, se encuentra el fortalecimiento de los sistemas de protección social (Ásgeirsdóttir et al., 2020; Van Hal, 2015).

Los resultados de este análisis de las tasas de mortalidad por suicidio dan cuenta de la necesidad de ampliar la disponibilidad de la información sobre defunciones comparable entre países a fin de completar la imagen del impacto de esta problemática en la región. Asimismo, se debe procurar la mejora de la calidad de la información estadística sobre suicidio y también sobre ideación suicida. Como se mencionó, factores culturales y religiosos pueden impactar la cobertura de la mortalidad por suicidio al existir presiones para certificar el fallecimiento como debido a otra causa, mientras se vuelve secundaria la debida a lesiones cuya intencionalidad queda no establecida, o más bien sin registro del mecanismo utilizado para provocar la muerte, contribuyendo con ello a un subregistro de la mortalidad por las lesiones autoinfligidas (Minayo et al., 2012). La ausencia de completitud de los datos estadísticos sobre suicidio representa un obstáculo para reconocer la verdadera magnitud de este problema social y de salud pública impidiendo, de igual manera, su visibilidad a cabalidad. Intervenciones destinadas a reducir el estigma que en ocasiones puede adscribirse a muertes por esta causa tendrían como efecto adicional la mejora del registro de suicidios (Sapag et al., 2018).

Si bien las informaciones tanto de mortalidad como de volúmenes poblaciones utilizadas en este análisis están homologadas por los criterios empleados por la Organización Mundial de la Salud (OMS) y Naciones Unidas, respectivamente, para incorporarlas en su base de datos o sus estimaciones, las posibilidades de un subregistro de las defunciones, el cual puede ser diferencial por países o grupos de edad, representan la principal limitación de este estudio. Otro aspecto que también constituye una limitación es el retraso en el reporte de la información sobre mortalidad que afecta lo oportuno de la misma. En el caso de México, por ejemplo, la diferencia temporal entre el registro y el reporte de las principales 
características de la mortalidad anual es de alrededor de 10 meses (Instituto Nacional de Estadística y Geografía, 2019). La demora de los países en reportar su información a la OMS afecta el número de naciones para las cuales es posible hacer un estudio comparativo como este, uniformando los periodos de análisis.

La ausencia de reducciones sustantivas en los niveles de mortalidad por suicidio en los grupos estudiados indica que este fenómeno no ha sido incorporado en el conjunto de acciones prioritarias en salud en general, ni en las de salud mental. El aumento de las tasas de mortalidad por suicidio, identificado para algunos grupos etarios en Chile, Cuba y Uruguay, da cuenta de la exacerbación de este problema y el posible empeoramiento de los factores que desencadenan o incentivan que los fallecimientos por esta causa acontezcan. El caso de Uruguay es especialmente preocupante debido a que la intensidad de la mortalidad por suicidio lo ubica, para 2015, como el doceavo país con la tasa más alta de defunciones masculinas por esta causa (31.4 defunciones masculinas por suicidio por 100,000 habitantes) de acuerdo al seguimiento del indicador 3.4.2 de los ODS. Esta cifra incluso excede las de países como Japón (27.4) o Estados Unidos (23.0) que suelen señalarse como sociedades en las que el suicidio acontece con mayor asiduidad (United Nations, 2020a). El suicidio ha sido señalado como un fenómeno que acaece con mayor frecuencia en las edades jóvenes y adultas (O'Connor et al., 2018; Rasmussen, Haavind y Dieserud, 2018), sin embargo, como los resultados de este análisis indican, las altas tasas de mortalidad por suicidio de adultos mayores apuntan la urgencia de emprender acciones integrales que involucren el mejoramiento de la salud mental y el acceso oportuno a servicios de apoyo para toda la población.

\section{Conclusiones e implicaciones}

Los diferenciales entre las tasas de mortalidad por suicidio de los ocho países analizados dan cuenta de las desigualdades registradas en la región para una causa de muerte prevenible. De igual forma, la estabilidad de las trayectorias de la mortalidad durante los once años estudiados traduce la ausencia de acciones específicas encaminadas a reducir su ocurrencia o bien la ineficacia de las existentes. La diversidad de las imágenes por grupo de edad resalta la importancia de examinar problemáticas como las del suicidio desglosadas por características centrales, así como con una perspectiva de contraste entre países. Los casos de Uruguay para las poblaciones joven, adulta y adulta mayor, de Cuba para adultos y adultos mayores y de Chile para adultos exige destinar acciones para dilucidar los 
factores que se asocian a patrones de mortalidad especialmente altos. Es importante reiterar la urgencia de mejorar la calidad de la información sobre el suicidio, la cual puede estar afectada por aspectos culturales y religiosos, así como por elementos de discriminación expresados como estigmas. De igual forma, se debe acentuar la relevancia de estructurar aproximaciones longitudinales que permitan identificar los cambios que en la salud mental puedan estarse gestando como resultado de enfermedades crónicas o no transmisibles como diabetes o cáncer. Las acciones preventivas específicamente dirigidas a determinar los riesgos de ideaciones suicidas y los intentos de suicidios conforman un espacio especialmente urgente para reducir la ocurrencia de esta causa de muerte prevenible.

La importancia de monitorear la mortalidad por suicidio como mecanismo para evidenciar, en su caso, los cambios en la salud mental de la población ha sido señalada en los ODS. Los resultados de este estudio podrían servir de punto de partida para el seguimiento que habrá de dar cuenta del cumplimiento de los compromisos de los ODS en 2030. Al mismo tiempo, podrán ser utilizados como referencia para medir el impacto que probablemente tendrá la pandemia de COVID-19 en la salud mental de las poblaciones de los países especialmente afectados por las vicisitudes sanitarias, económicas y sociales que esta ha provocado.

\section{Referencias bibliográficas}

Arias Vázquez, P. I., Castillo Avila, R. G., Dominguez Zentella, M., HernándezDíaz, Y., González-Castro, T. B., Tovilla-Zárate, C. A., Juárez-Rojop, I. E., López-Narváez, M. L. y Frésan, A. (2018). Prevalence and correlations between suicide attempt, depression, substance use, and functionality among patients with limb amputations. International Journal of Rehabilitation Research, 47(1), 52-56. https://doi.org/10.1097/MRR.0000000000000259

Ásgeirsdóttir, H. G., Valdimarsdóttir, U. A., Nyberg, U.,Lund, S. H., Tomasson, G., Dorsteinsdóttir, D. K., Ásgeirsdóttir, T. L. y Hauksdóttir, A. (2020). Suicide rates in Iceland before and after the $2008 \mathrm{Global}$ Recession: a nationwide population-based study. European Journal of Public Health, 30(6), 1102-1108. https://doi.org/10.1093/eurpub/ckaa121

Bach, S. L., Molina, M., Jansen, K., da Silva, R. A. y Souza, L. (2018). Suicide risk and childhood trauma in individuals diagnosed with posttraumatic stress disorder. Trends in Psychiatry and Psychotherapy, 40(3), 253-257. https://doi.org/10.1590/2237-6089-2017-0101 
Bachmann, S. (2018). Epidemiology of Suicide and the Psychiatric Perspective. International Journal of Environmental Research and Public Health, 15(7), 1425. https://doi.org/10.3390/ijerph15071425

Bernert, R. A., Kim, J. S., Iwata, N. G. y Perlis, M. L. (2015). Sleep disturbances as an evidence-based suicide risk factor. Current Psychiatry Reports, 17(3), 554. https://doi.org/10.1007/s11920-015-0554-4

Bustamante, F., Ramirez, V., Urquidi, C., Bustos, V., Yaseen, Z. y Galynker, I. (2016). Trends and Most Frequent Methods of Suicide in Chile Between 2001 and 2010. Crisis, 37(1), 21-30. https://doi.org/10.1027/02275910/a000357

Calati, R., Ferrari, C., Brittner, M., Oasi, O., Olié, E., Carvalho, A. F. y Courtet, P. (2019). Suicidal thoughts and behaviors and social isolation: A narrative review of the literature. Journal of Affective Disorders, 245, 653-667. https://doi.org/10.1016/j.jad.2018.11.022

Carmel, A., Ries, R., West, I. I., Bumgardner, K. y Roy-Byrne, P. (2016). Suicide risk and associated demographic and clinical correlates among primary care patients with recent drug use. The American Journal of Drug and Alcohol Abuse, 42(3), 351-357. https://doi.org/10.3109/ 00952990.2015 .1133634

Cecchini, S., Filgueira, F. y Robles, C., (2014). Sistemas de protección social en América Latina y el Caribe. Una perspectiva comparada. Serie Políticas Sociales 202. Santiago de Chile: CEPAL; Ministerio de Asuntos Exteriores de Noruega. https://www.cepal.org/es/ publicaciones/36831-sistemas-proteccion-social-america-latinacaribe-perspectiva-comparada

Cendales, R., Vanegas, C., Fierro, M., Córdoba, R. y Olarte, A. (2007). Tendencias del suicidio en Colombia, 1985-2002. Revista Panamericana de Salud Pública, 22(4), 231-238. https://doi. org/10.1590/s1020-49892007000900002

Chan, C. H., Caine, E. D., You, S., Fu, K. W., Chang, S. S. y Yip, P. S. (2014). Suicide rates among working-age adults in South Korea before and after the 2008 economic crisis. Journal of Epidemiology \& Community Health, 68(3), 246-252. https://doi.org/10.1136/ jech-2013-202759

Chang, S. S., Stuckler, D., Yip, P. y Gunnell, D. (2013). Impact of 2008 global economic crisis on suicide: time trend study in 54 countries. BMJ, 347, f5239. https://doi.org/10.1136/bmj.f5239 
Chastang, F., Rioux, P., Dupont, I., Baranger, E., Kovess, V.y Zarifian, E. (1998). Suicide attempts and job insecurity: a complex association. European Psychiatry, 13(7), 359-364. https://doi.org/10.1016/ So924-9338(99)80703-2

Comisión Económica para América Latina y el Caribe. (2020). Enfrentar los efectos cada vez mayores del COVID-19 para una reactivación con igualdad: nuevas proyecciones (Informe Especial COVID-19 No. 5).https://www.cepal.org/es/publicaciones/45782-enfrentarefectos-cada-vez-mayores-covid-19-reactivacion-igualdad-nuevas

Dubey, M. J., Ghosh, R., Chatterjee, S., Biswas, P., Chatterjee, S. y Dubey, S. (2020). COVID-19 and addiction. Diabetes \& Metabolic Syndrome: Clinical Research \& Reviews, 14(5), 817-823. https://doi.org/10.1016/j. dsx.2020.06.008

Fahey, R. A., Matsubayashi, T. y Ueda, M. (2018). Tracking the Werther Effect on social media: Emotional responses to prominent suicide deaths on twitter and subsequent increases in suicide. Social Science \& Medicine, 219, 19-29. https://doi.org/10.1016/j.socscimed.2018.10.004

Fang, F., Fall, K., Mittleman, M. A., Sparén, P., Ye, W., Adami, H. O. y Valdimarsdóttir, U. (2012). Suicide and Cardiovascular Death After a Cancer Diagnosis. The New England Journal of Medicine, 366(14), 1310-1318. https://doi.org/10.1056/NEJMoa1110307

Felitti, V. J., Anda, R. F., Nordenberg, D., Williamson, D. F., Spitz, A. M., Edwards, V., Koss, M. P. y Marks, J. S. (2019). Reprint of: Relationship of Childhood Abuse and Household Dysfunction to Many of the Leading Causes of Death in Adults: The Adverse Childhood Experiences (ACE) Study. American Journal of Preventive Medicine, 56(6), 774-786. https://doi.org/10.1016/j.amepre.2019.04.001

Ghanbari, B., Malakouti, S. K., Nojomi, M., De Leo, D. y Saeed, K. (2015). Alcohol Abuse and Suicide Attempt in Iran: A Case-Crossover Study. Global journal of health science, 8(7), 58-67. https://doi.org/10.5539/gjhs. v8n7p58

Gómez-Peralta, T. G., González-Castro, T. B., Fresan, A., Tovilla-Zárate, C. A., Juárez-Rojop, I. E., Villar-Soto, M., Hernández-Díaz, Y., LópezNarváez, M. L., Ble-Castillo, J. L., Pérez-Hernández, N. y RodríguezPérez, J. M. (2018). Risk Factors and Prevalence of Suicide Attempt in Patients with Type 2 Diabetes in the Mexican Population. International Journal of Environmental Research and Public Health, 15(6), 1198. https://doi.org/10.3390/ijerph15061198 
Håkansson A. (2020). Changes in Gambling Behavior during the COVID-19 Pandemic-A Web Survey Study in Sweden. International Journal of Environmental Research and Public Health, 17(11), 4013. https:// doi.org/10.3390/ijerph17114013

ICD-10. (2019). International Statistical Classification of Diseases and Related Health Problems 10th Revision. World Health Organization. https://icd.who.int/browse10/2019/en

Instituto Nacional de Estadística y Geografía (2019, 29 de octubre). Características de las defunciones registradas en México durante 2019 (Comunicado de prensa No. 480/20).

Klaassen, Z., Arora, K., Wilson, S. N., King, S. A., Madi, R., Neal Jr., D. E., Kurdyak, P., Kulkarni, G. S., Lewis, R. W. y Terris, M. K. (2018). Decreasing suicide risk among patients with prostate cancer: Implications for depression, erectile dysfunction, and suicidal ideation screening. Urologic Oncology: Seminars and Original Investigations, 36(2), 60-66. https://doi.org/10.1016/j.urolonc.2017.09.007

Klonsky, E. D., May, A. M. y Saffer, B. Y. (2016). Suicide, Suicide Attempts, and Suicidal Ideation. Annual Review of Clinical Psychology, 12, 307330. https://doi.org/10.1146/annurev-clinpsy-021815-093204

Kohn, R., Levav, I., de Almeida, J. M., Vicente, B., Andrade, L., CaraveoAnduaga, J. J., Saxena, S. y Saraceno, B. (2005). Los trastornos mentales en América Latina y el Caribe: asunto prioritario para la salud pública. Revista Panamericana de Salud Pública, 18(4-5), 229-240. https://doi.org/10.1590/s1020-49892005000900002

Laanani, M., Ghosn, W., Jougla, E. y Rey, G. (2015). Impact of unemployment variations on suicide mortality in Western European countries (2000-2010). Journal of Epidemiology \& Community Health, 69(2), 103-109. https://doi.org/10.1136/jech-2013-203624

Love, H. A., Nalbone, D. P., Hecker, L. L., Sweeney, K. A. y Dharnidharka, P. (2018). Suicidal Risk Following the Termination of Romantic Relationships. Crisis, 39(3), 166-174. https://doi.org/10.1027/02275910/a000484

Mann, J. J. y Michel, C. A. (2016). Prevention of Firearm Suicide in the United States: What Works and What Is Possible. The American Journal of Psychiatry, 173(10), 969-979. https://doi.org/10.1176/appi. ajp.2016.16010069 
Martini, M., da Fonseca, R. C., de Sousa, M. H., de Azambuja Farias, C., Cardoso, T. A., Kunz, M., Longaray, V. K. y Magalhães, P. (2019). Age and sex trends for suicide in Brazil between 2000 and 2016. Social Psychiatry and Psychiatric Epidemiology, 54(7), 857-860. https:// doi.org/10.1007/s00127-019-01689-8

Mascayano, F., Irrazabal, M., D Emilia, W., Vaner, S. J., Sapag, J. C., Alvarado, R., Yang, L. H.y Sinah, B. (2015). Suicide in Latin America: a growing public health issue. Revista de la Facultad de Ciencias Médicas de Cordoba, 72(4), 295-303. https://doi.org/10.31053/1853.0605.v72. n4.13837

Mascayano, F., Tapia, T., Schilling, S., Alvarado, R., Tapia, E., Lips, W. y Yang, L. H. (2016). Stigma toward mental illness in Latin America and the Caribbean: a systematic review. Revista Brasileira de Psiquiatria, 38(1), 73-85. https://doi.org/10.1590/1516-4446-2015-1652

Merzagora, I., Mugellini, G., Amadasi, A. y Travaini, G. (2016). Suicide Risk and the Economic Crisis: An Exploratory Analysis of the Case of Milan. PloS One, 17(12), e0166244. https://doi.org/10.1371/journal. pone.0166244

Milner, A., Morrell, S. y LaMontagne, A. D. (2014). Economically inactive, unemployed and employed suicides in Australia by age and sex over a 10-year period: what was the impact of the 2007 economic recession? International Journal of Epidemiology, 43(5), 1500-1507. https://doi.org/10.1093/ije/dyu148

Min, K. B., Park, S. G., Hwang, S. H. y Min, J. Y. (2015). Precarious employment and the risk of suicidal ideation and suicide attempts. Preventive Medicine, 71, 72-76. https://doi.org/10.1016/j.ypmed.2014.12.017

Minayo, M. C., Pinto, L. W., Assis, S. G., Cavalcante, F. G. y Mangas, R. M. (2012). Trends in suicide mortality among Brazilian adults and elderly, 1980-2006. Revista de Saúde Pública, 46(2), 300-309. https://doi. org/10.159o/s0034-89102012000200012

Naghavi, M. y Global Burden of Disease Self-Harm Collaborators. (2019). Global, regional, and national burden of suicide mortality 1990 to 2016: systematic analysis for the Global Burden of Disease Study 2016. BMJ, 364, 194. https://doi.org/10.1136/bmj.194

Nordt, C., Warnke, I., Seifritz, E. y Kawohl, W. (2015). Modelling suicide and unemployment: a longitudinal analysis covering 63 countries, 2000-11. The Lancet. Psychiatry, 2(3), 239-245. https://doi. org/10.1016/S2215-0366(14)00118-7 
Núñez-Samudio, V., Jiménez-Domínguez, A., López Castillo, H. y Landires, I. (2020). Epidemiologic Characteristics of Suicide in Panama, 2007-2016. Medicina, 56(9), 442. https://doi.org/10.3390/ medicina56090442

O'Connor, R. C., Wetherall, K., Cleare, S., Eschle, S., Drummond, J., Ferguson, E., O'Connor, D. B. y O'Carroll, R. E. (2018). Suicide attempts and non-suicidal self-harm: national prevalence study of young adults. BJPsych open, 4(3), 142-148. https://doi.org/10.1192/bjo.2018.14

Oliffe, J. L., Rossnagel, E., Seidler, Z. E., Kealy, D., Ogrodniczuk, J. S. y Rice, S. M. (2019). Men's Depression and Suicide. Current Psychiatry Reports, 21(10), 103. https://doi.org/10.1007/s11920-019-1088-y

Orellana, J. D., Balieiro, A. A., Fonseca, F. R., Basta, P. C. y Souza, M. L. (2016). Spatial-temporal trends and risk of suicide in Central Brazil: an ecological study contrasting indigenous and non-indigenous populations. Revista Brasileira de Psiquiatria, 38(3), 222-230. https://doi.org/10.1590/1516-4446-2015-1720

Organización Panamericana de la Salud. (2014). Mortalidad por suicidio en las Américas (Informe regional, Washington, D. C.).

Ortiz-Prado, E., Simbaña, K., Gómez, L., Henriquez-Trujillo, A. R., CornejoLeon, F., Vasconez, E., Castillo, D. y Viscor, G. (2017). The disease burden of suicide in Ecuador, a 15 years' geodemographic crosssectional study (2001-2015). BMC Psychiatry, 17(1), 342. https://doi. org/10.1186/s12888-017-1502-O

Osazuwa-Peters, N., Simpson, M. C., Zhao, L., Boakye, E. A., Olomukoro, S. I., Deshields, T., Loux, T. M., Varvares, M. A. y Schootman, M. (2018). Suicide risk among cancer survivors: Head and neck versus other cancers. Cancer, 124(20), 4072-4079. https://doi.org/10.1002/ cncr.31675

Otzen, T., Sanhueza, A., Manterola, C. y Escamilla-Cejudo, J. A. (2014). Mortalidad por suicidio en Chile: tendencias en los años 1998-2011. Revista médica de Chile, 142(3), 305-313. https://doi.org/10.4067/ S0034-98872014000300004

Owen-Smith, A. A., Ahmedani, B. K., Peterson, E., Simon, G. E., Rossom, R. C., Lynch, F. L., Lu, C. Y., Waitzfelder, B. E., Beck, A., DeBar, L. L., Sanon, V., Maaz, Y., Khan, S., Miller-Matero, L. R., Prabhakar, D., Frank, C., Drake, C. L. y Braciszewski, J. M. (2019). The Mediating Effect of Sleep Disturbance on the Relationship Between Nonmalignant Chronic Pain and Suicide Death. Pain Practice, 19(4), 382-389. https://doi.org/10.1111/papr.12750 
Pérez-Fuentes, G., Olfson, M., Villegas, L., Morcillo, C., Wang, S. y Blanco, C. (2013). Prevalence and correlates of child sexual abuse: a national study. Comprehensive Psychiatry, 54(1), 16-27. https://doi. org/10.1016/j.comppsych.2012.05.010

Pigeon, W. R., Pinquart, M. y Conner, K. (2012). Meta-analysis of sleep disturbance and suicidal thoughts and behaviors. The Journal of Clinical Psychiatry, 73(9), e1160-e1167. https://doi.org/10.4088/ JCP.11r07586

Plöderl, M., Wagenmakers, E. J., Tremblay, P., Ramsay, R., Kralovec, K., Fartacek, C. y Fartacek, R. (2013). Suicide risk and sexual orientation: a critical review. Archives of Sexual Behavior, 42(5), 715-727. https:// doi.org/10.1007/s10508-012-0056-y

Pompili, M., Lester, D., Forte, A., Seretti, M. E., Erbuto, D., Lamis, D. A., Amore, M. y Girardi, P. (2014). Bisexuality and suicide: a systematic review of the current literature. The Journal of Sexual Medicine, 17(8), 1903-1913. https://doi.org/10.1111/jsm.12581

Pritchard, C. y Hean, S. (2008). Suicide and undetermined deaths among youths and young adults in Latin America: comparison with the 10 major developed countries - a source of hidden suicides? Crisis, 29(3), 145-153. https://doi.org/10.1027/0227-5910.29.3.145

Rasmussen, M. L., Haavind, H.y Dieserud, G. (2018). Young Men, Masculinities, and Suicide. Archives of Suicide Research, 22(2), 327-343. https:// doi.org/10.1080/13811118.2017.1340855

Rice, S. M., Oliffe, J. L., Kealy, D., Seidler, Z. E. y Ogrodniczuk, J. S. (2020). Men's Help-Seeking for Depression: Attitudinal and Structural Barriers in Symptomatic Men. Journal of Primary Care \& Community Health. https://doi.org/10.1177/2150132720921686

Sapag, J. C., Sena, B. F., Bustamante, I. V., Bobbili, S. J., Velasco, P. R., Mascayano, F., Alvarado, R. y Khenti, A. (2018). Stigma towards mental illness and substance use issues in primary health care: Challenges and opportunities for Latin America. Global Public Health, 13(10), 1468-1480. https://doi.org/10.1080/17441692.2017.13 56347

Saurina, C., Bragulat, B., Saez, M. y López-Casasnovas, G. (2013). A conditional model for estimating the increase in suicides associated with the 2008-2010 economic recession in England. Journal of Epidemiology \& Community Health, 67(9), 779-787. https://doi. org/10.1136/jech-2013-202645 
Spokas, M., Wenzel, A., Stirman, S. W., Brown, G. K. y Beck, A. T. (2009). Suicide risk factors and mediators between childhood sexual abuse and suicide ideation among male and female suicide attempters. Journal of Traumatic Stress, 22(5), 467-470. https:// doi.org/10.1002/jts.20438

Stanley, I. H., Boffa, J. W., Rogers, M. L., Hom, M. A., Albanese, B. J., Chu, C., Capron, D. W., Schmidt, N. B. y Joiner, T. E. (2018). Anxiety sensitivity and suicidal ideation/suicide risk: A meta-analysis. Journal of Consulting and Clinical Psychology, 86(11), 946-960. https://doi. org/10.1037/ccpooo0342

Sun, Y., Li, Y., Bao, Y., Meng, S., Sun, Y., Schumann, G., Kosten, T., Strang, J., Lu, L. y Shi, J. (2020). Brief Report: Increased Addictive Internet and Substance Use Behavior During the COVID-19 Pandemic in China. The American Journal on Addictions, 29(4), 268-270. https://doi. org/10.1111/ajad.13066

Tang, N. K. y Crane, C. (2006). Suicidality in chronic pain: a review of the prevalence, risk factors and psychological links. Psychological Medicine, 36(5), 575-586. https://doi.org/10.1017/So033291705006859

Teti, G. L., Rebok, F., Rojas, S. M., Grendas, L. y Daray, F. M. (2014). Systematic review of risk factors for suicide and suicide attempt among psychiatric patients in Latin America and Caribbean. Revista Panamericana de Salud Pública, 36(2), 124-133. https://www. scielosp.org/article/rpsp/2014.v36n2/124-133/

Till, B., Tran, U. S. y Niederkrotenthaler, T. (2017). Relationship Satisfaction and Risk Factors for Suicide. Crisis, 38(1), 7-16. https://doi. org/10.1027/0227-5910/a000407

United Nations. (2020a). SDG Monitoring and Reporting Toolkit for UN Country Teams. Department of Economic and Social Affairs. Statistics Division. Sustainable Development Goals. https://unstats. un.org/sdgs/unct-toolkit/

United Nations. (2020b). World Population Prospects 2019. Department of Economic and Social Affairs. Population Dynamics. Population by age and sex (thousands). https://population.un.org/wpp/ DataQuery/

Van Hal, G. (2015). The true cost of the economic crisis on psychological well-being: a review. Psychology Research and Behavior Management, 8, 17-25. https://doi.org/10.2147/PRBM.S44732 
Vigo, D., Jones, L., Maidana, D. E., Krausz, M. y Kestel, D. (2018). Carga de los trastornos mentales, neurológicos, por consumo de sustancias y suicidio y prioridades de salud mental en Argentina. Vertex, 29(142), 304-312. http://www.editorialpolemos.com.ar/vertex142. php

World Health Organization. (2020). WHO Mortality Database. https://www. who.int/data/data-collection-tools/who-mortality-databasehttps:// apps.who.int/healthinfo/statistics/mortality/causeofdeath_query/ start.php

Yuodelis-Flores, C. y Ries, R. K. (2015). Addiction and suicide: A review. The American Journal on Addictions, 24(2), 98-104. https://doi. org/10.1111/ajad.12185 